\title{
Appropriateness of Pediatric Hospitalization in a General Hospital in Kuwait
}

\author{
Magdy H. Shafik Tarek M.M. Seoudi Tarek S. Raway Nowair Z. Al Harbash \\ Meshal M.A. Ahmad Hanan F. Al Mutairi \\ Department of Pediatrics, Farwania Hospital, Kuwait City, Kuwait
}

\section{Key Words}

Appropriateness $\cdot$ Admission $\cdot$ Pediatric

\begin{abstract}
Objectives: To determine the rate of inappropriate pediatric admissions using the Pediatric Appropriateness Evaluation Protocol (PAEP) and to examine variables associated with inappropriateness of admissions. Subjects and Methods: A prospective study was conducted in the Department of Pediatrics, Farwania General Hospital, Kuwait, to examine successive admissions for appropriateness of admission as well as several sociodemographic characteristics over a 5-month period (August 2010 to December 2010). A total of 1,022 admissions were included. Results: Of the 1,022 admissions, 416 (40.7\%) were considered inappropriate. Factors associated with a higher rate of inappropriate admission included older age of patients and self-referral. Conclusion: The rate of inappropriate hospitalization of children was high in Farwania Hospital, Kuwait, probably due to the relatively free health care services, parental preference for hospital care, easy access to hospital services, and insufficient education about the child's condition.

Copyright $\odot 2012$ S. Karger AG, Basel
\end{abstract}

\section{Introduction}

Kuwait is a developing nation with an increasing population and an increasing demand for medical services. Over the last few decades, the expansion of medical services and particularly of hospital-based services has been remarkable. However, this expansion may not continue indefinitely, and optimizing the use of the current services should become the goal in the near future. Thus, the efficient use of hospital resources that help cut the expenses without compromising the quality of care provided for children should be among the goals of those who provide medical services for children in Kuwait [1].

Using evaluation protocols, such as the Appropriateness Evaluation Protocol (AEP), international studies have shown that $10-30 \%$ of hospital admissions are medically unnecessary $[1,2]$. The AEP was first designed and developed in the USA by Gertman and Restuccia [3] for assessing potentially unnecessary hospital days of care. Twenty-seven objective criteria items related to medical services, nursing/life support services, and patient condition factors were incorporated into the protocol.

The pediatric version of the AEP (PAEP) (appendix) was derived from the original AEP by Kemper [4]. The PAEP is one of the most widely used instruments for assessing inappropriate hospital use for children, and the validity and reliability of the PAEP have been tested several 


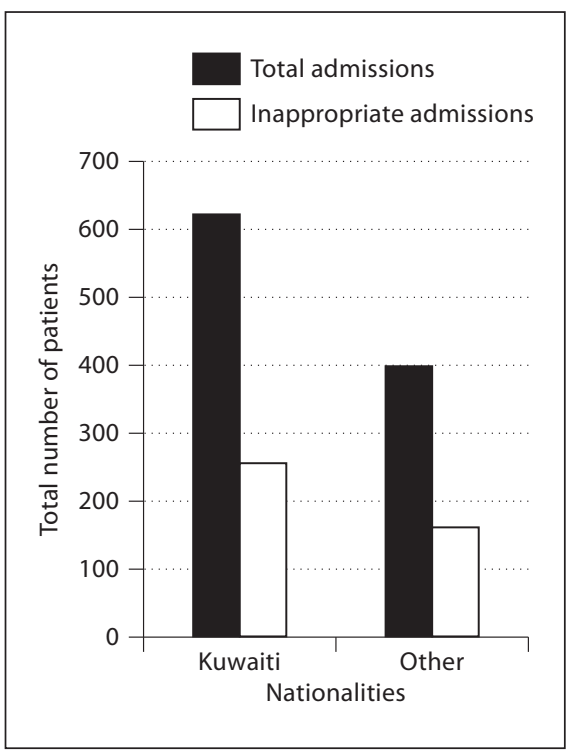

Fig. 1. Distribution of inappropriate admissions by nationality (in comparison to total admissions).

times $[5,6]$. Hence, the objective of this study was to use the PAEP to determine factors associated with the appropriateness of pediatric hospitalization in a general hospital.

\section{Subjects and Methods}

Farwania Hospital is a general hospital which serves a population of approximately 900,000 . An average of 380 children under 12 years of age visited the Pediatric Casualty Department daily in the year 2010. The average daily admission to Pediatric Wards was 9 patients.

A prospective, descriptive study was carried out in 1,022 pediatric patients (530 males and 492 females; age range from birth to 12 years) admitted to the Department of Pediatrics of Farwania Hospital over a period of 5 months from August to December 2010. Babies born in the hospital who were admitted to the special care unit were excluded. Of the 1,022 children included in the study, 624 were Kuwaiti and 398 were of other nationalities. Several items such as age, sex, nationality, time of admission, and type of referral were analyzed using the Statistical Package for the Social Sciences program (SPSS). Of the 1,022 patients, 343 were randomly selected; their medical records were examined and further analyzed for outcome and follow-up arrangements.

Age was divided into three strata: $\leq 1$ year, $>1$ year but $\leq 5$ years, and $>5$ years. The patients were assigned to each age strata as follows: $\leq 1$ year, $n=412 ;>1$ year but $\leq 5$ years, $n=378$, and $>5$ years, $\mathrm{n}=232$. Time of admission was divided into 3 categories: morning and early afternoon (7:00 a.m. to 1:59 p.m.), afternoon (2:00 p.m. to 9:59 p.m.), and night (10:00 p.m. to 6:59 a.m.). The admitted children presented to the Pediatric Casualty Department as self-refer- rals, referrals from primary care clinics, or other forms of referral (from other hospitals, other departments in the same hospital, or private clinics). Upon discharge, children were classified as follows: those who needed outpatient follow-up, those who did not need further follow-up, those who were discharged against medical advice, those who were referred to other departments or other hospitals, and those who died. Case diagnosis was not subjected to analysis as part of the study since the appropriateness of admission was evaluated independently of the diagnosis. However, the diagnosis was further analyzed for possible insight as to the causes of inappropriate admission and suggestions for ways to reduce such admissions.

\section{Results}

Of the 1,022 patients, $416(40.7 \%)$ were considered to be inappropriately admitted based on the PAEP. Of those 416 patients, 136 (32.7\%) were $\leq 1$ year old; 177 (42.5\%) were $>1$ year old but $\leq 5$ years old, and $103(24.8 \%)$ were $\geq 5$ years old. Group comparisons revealed that inappropriate admission was less in children younger than 1 year of age $(136 / 412 ; 33 \%)$ than in the other 2 groups $(177 / 378$, $46.8 \% ; 103 / 232,44.3 \%)$, and the difference was statistically significant $(\mathrm{p}<0.001)$.

Two hundred seventeen (40.9\%) of the 530 male patients and 199 (40.4\%) of the 492 female patients were classified as inappropriate admissions, and the difference was not statistically significant $(\mathrm{p}>0.001)$. Two hundred fifty-five (40.8\%) of the 624 Kuwaitis and 161 (40.4\%) of the 398 non-Kuwaitis were considered inappropriate admissions (fig. 1), and the difference was not statistically significant. Among the children who were admitted in the morning and early afternoon, 122/314 (38.9\%) were inappropriate admissions; 160/391 (40.9\%) afternoon admissions and 134/317 (42.2\%) night admissions were also inappropriate admissions. The differences were not statistically significant $(\mathrm{p}>0.001)$.

Of the 1,022 patients, 931 (91\%) were admitted to the Casualty Department on a self-referral basis. In those 931, the rate of inappropriate admission was $40.8 \%$. Among 44 patients who were referred from primary care, the rate of inappropriate admission was $34 \%$ (15/44). This was significantly lower than the rate of inappropriate admission in the self-referred group (table 1).

Among the 343 patients randomly selected for further analysis, 135 patients were considered inappropriately admitted (39.4\%), 136 (39.65\%) were discharged with follow-up in the Outpatient Clinic at later dates; 139 (40.52\%) were discharged without further follow-up, 53 (15.45\%) were discharged against medical advice without further follow-up, 10 (2.92\%) were referred to other hospitals, and $5(1.46 \%)$ were referred to other departments within the 


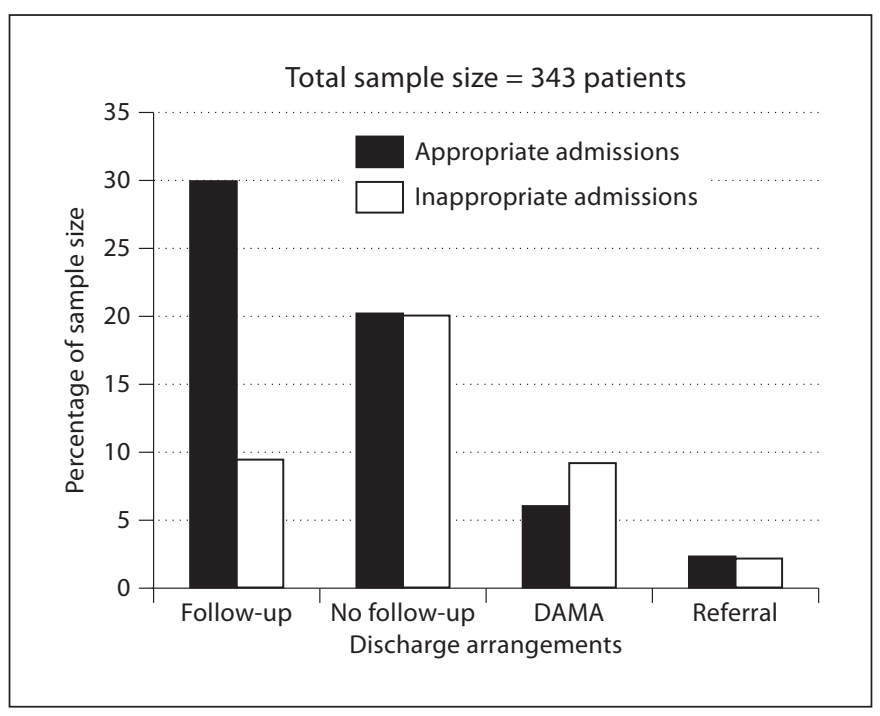

Fig. 2. Percentage distribution of discharge arrangement for both appropriate and inappropriate admissions. DAMA = Discharge against medical advice.

Table 1. Pediatric hospital admissions $(1,022)$ according to specified characteristics

\begin{tabular}{|c|c|c|}
\hline Characteristic & $\begin{array}{l}\text { Admissions, } \\
\mathrm{n}(\%)\end{array}$ & $\begin{array}{l}\text { Admissions } \\
\text { evaluated as } \\
\text { inappropriate, } \\
\mathrm{n} / \mathrm{N}(\%)\end{array}$ \\
\hline \multicolumn{3}{|l|}{ Age } \\
\hline$<1$ year & $412(40.3)$ & $136 / 412(33)$ \\
\hline $1-5$ years & $378(37)$ & $177 / 378(46.8)$ \\
\hline$>5$ years & $232(22.6)$ & $103 / 232(44.3)$ \\
\hline \multicolumn{3}{|l|}{ Sex } \\
\hline Male & $530(51.9)$ & $217 / 530(40.9)$ \\
\hline Female & $492(48.1)$ & $199 / 492(40.4)$ \\
\hline Total & $1,022(100)$ & $416 / 1,022(40.7)$ \\
\hline \multicolumn{3}{|l|}{ Nationality } \\
\hline Kuwaiti & $624(61)$ & $255 / 624(40.8)$ \\
\hline Other & $398(39)$ & $161 / 398(40.4)$ \\
\hline \multicolumn{3}{|l|}{ Time of admission } \\
\hline Morning $^{1}$ & $314(30.7)$ & $122 / 314(38.9)$ \\
\hline Afternoon ${ }^{2}$ & $391(38.3)$ & $160 / 391(40.9)$ \\
\hline Night $^{3}$ & $317(31)$ & $134 / 317(42.2)$ \\
\hline \multicolumn{3}{|l|}{ Type of referral } \\
\hline Self-referral & $931(91.1)$ & $380 / 93(140.8)$ \\
\hline Primary care & $44(4.3)$ & $15 / 44(34)$ \\
\hline Others & $47(4.06)$ & $21 / 47(44.6)$ \\
\hline
\end{tabular}

${ }^{1}$ 7:00 a.m. to 1:59 p.m. ${ }^{2}$ 2:00 p.m. to 9:59 p.m. ${ }^{3} 10: 00$ p.m. to $6: 59$ a.m. $\mathrm{N}=$ Total number of cases of the specified characteristic; $\mathrm{n}=$ number of cases evaluated as inappropriate admission by the PAEP. hospital (fig. 2). Of the 136 patients discharged to followup in the Outpatient Clinic, 33 (24.3\%) were considered inappropriately admitted.

The ten most common diagnoses at the time of admission and the number of patients admitted with each diagnosis are given in (table 2), as are the corresponding number and percentage of inappropriate admissions in each diagnosis. The inappropriate admission rate was high when the diagnosis was: gastroenteritis, 75/113 (64.6\%); seizures, 53/79 (65.8\%); upper respiratory tract infection, $31 / 38$ (81.5\%), or vomiting, $20 / 33(66 \%)$. The rate of inappropriate admission was less when the diagnosis was: chest infection, 56/184 (30.4\%); acute bronchiolitis, 30/102 (29.4\%), or urinary tract infection, 42/151 (27.8\%).

\section{Discussion}

The results of this study showed that the rate of medically inappropriate admission at $40.7 \%$ was higher than that of other countries; $24 \%$ was reported for Canada [6] and Australia [7] and 10-30\% was reported for Europe, the USA, Israel, and Italy $[1,4,8,9]$. These differences could be attributed to the country's health care system based on whether or not health care is provided free of charge by the government or paid by individuals through private insurance. In Kuwait, the Kuwaitis are provided free health services in the public sector while non-Kuwaitis pay a small fixed amount. The relatively free health care system in Kuwait could account for higher inappropriate pediatric hospitalization rates in Kuwait compared to other countries.

The inappropriate admissions rate of 33\% among patients $\leq 1$ year old compared to $44.3 \%$ in patients $\geq 5$ years of age could reflect the difference in the complexity of diagnoses between infants and older children. Infants are most likely to be hospitalized for prematurity, congenital problems, or infectious illnesses, all conditions that would lead to intensive medical services, whereas older children present with more chronic diseases and may be hospitalized for investigations and less intensive therapy.

There was no difference in the rate of inappropriate admission at different times of the day, contrary to the findings of other studies $[9,10]$. In the study by Bianco et al. [9], inappropriate admission occurred more during the day and was interpreted by use of the hospital as a primary care facility because it was easier to attend and find a pediatrician during the day [9]. 
Table 2. Distribution of clinical diagnoses for all admissions and inappropriate admissions

\begin{tabular}{lcc}
\hline Admission diagnosis & $\begin{array}{l}\text { All admissions, } \\
\mathrm{n}(\%)\end{array}$ & $\begin{array}{l}\text { Inappropriate } \\
\text { admissions, } \\
\mathrm{n}(\%)^{\mathrm{a}}\end{array}$ \\
\hline Chest infection $^{\mathrm{b}}$ & $184(18.0)$ & $56(13.46)$ \\
Bronchial asthma $_{\text {Urinary tract infection }}$ & $164(16.05)$ & $61(14.66)$ \\
Gastroenteritis & $151(14.77)$ & $42(10.09)$ \\
Acute bronchiolitis & $113(11.06)$ & $75(18.02)$ \\
Seizures (mostly febrile convulsions) & $79(7.73)$ & $53(12.74)$ \\
Fever (including neonatal fever) & $61(5.97)$ & $25(6.02)$ \\
Upper respiratory tract infection & $38(3.72)$ & $31(7.45)$ \\
Vomiting (no other findings) & $33(3.32)$ & $20(4.82)$ \\
Neonatal jaundice & $30(2.94)$ & $0(0.00)$ \\
Other diagnosis & $67(6.56)$ & $23(5.53)$ \\
\hline Total & $1,022(100)$ & $416(100)$ \\
\hline
\end{tabular}

${ }^{a}$ Percentage of inappropriate admissions is calculated out of the total number of inappropriate admissions (416). ${ }^{\mathrm{b}}$ Other than acute bronchiolitis.

However, in Kuwait, access to hospital pediatricians is possible all day long although access to primary care facilities is also available. Parental preference for hospital care, particularly during an acute illness, is apparently a factor that favors inappropriate admission.

Also, in our study sex did not have any effect on the rate of inappropriate admission, contrary to other studies [8-10]. This can be explained by the fact that almost overall equal numbers of both sexes were referred to the emergency room and this can be interpreted as equal care given by parents and caregivers to both boys and girls in Kuwait.

In the group of 343 patients that was randomly selected, the number of children who were scheduled for follow-up in the outpatient department was significantly higher in those who were considered to be appropriately admitted than in those who were considered to be inappropriately admitted (49.5 vs. $24.4 \%)$. This was to be expected; however, what is significant is that $24.4 \%$ of the children who were considered inappropriately admitted needed follow-up, emphasizing that the lack of need to admit to hospital does not necessarily mean the lack of need to treat, investigate, and follow-up.

Inappropriate hospitalization not only has an economic impact on the healthcare system but it could also have a psychological and physical impact on the child and the child's family, probably because several events could make the hospital stay a potentially stressful experience for children. Hence, unnecessary hospitalizations should be avoided for routine procedures as well as for chronic illnesses [11].

Acute and chronic respiratory diseases represent a large proportion of pediatric hospital admissions, both appropriate and inappropriate $34 \%$ of the total admissions in our study; $33.6 \%$ of them were considered inappropriately admitted) (table 2). Modern technology in the field of respiratory treatment, such as oxygen concentrators, suction and humidification machines, and nebulization equipment, allows for home treatment to be both effective and feasible for some of the respiratory diseases that are currently treated in hospital and for prolonged periods. Intravenous therapy can be administered on an outpatient basis or as home therapy. Innovations in antibiotic therapy, such as once-a-day dosage and technological innovation in infusion pumps, are some of the valuable ways to decrease the need for hospital admission. To allow for safe home nursing, parents should be trained to cope with the needs of their child at home, and home nursing facilities should be provided. Training may include nasogastric feeding, intramuscular injections, management of central venous lines, tracheostomy care, and home glucose monitoring [12].

Many pediatric hospitalizations might be avoided if parents and children were better educated about the child's condition, medications, the need for follow-up care, and the importance of avoiding known disease triggers [11]. Based on our findings, we believe that establishing an area for short-term observation in the emergency room that can provide intravenous hydration and/or observation of the response to treatment for children with acute respiratory problems (e.g. bronchial asthma or croup) might also help to lower the rate of unnecessary hospitalization. It is possible that improving these items will cause the rate of unnecessary admissions to decrease, so we can provide efficient use of hospital resources and cut the expenses without compromising the quality of care provided for children.

\section{Conclusion}

The rate of inappropriate hospitalization of children was high in Farwania Hospital, Kuwait. This could be explained by the relatively free health care services, parental preference for hospital care, easy access to the hospital services, and insufficient education about the child's condition. 


\section{Appendix}

Pediatric Appropriateness Evaluation Protocol

1 Surgery or procedure scheduled within $24 \mathrm{~h}$ necessitating:

(A) General or regional anesthesia

(B) Use of equipment, facilities, or procedures available only in a hospital

2 Treatment in an intensive care unit

3 Vital sign monitoring every $2 \mathrm{~h}$ or more often (may include telemetry or bedside cardiac monitor)

4 Intravenous medications and/or fluid replacement (does not include tube feedings)

5 Chemotherapeutic agents that require continuous observation for life-threatening toxic reaction

6 Intramuscular antibiotics at least every $8 \mathrm{~h}$

7 Continuous or intermittent nebulizer use at least every $4 \mathrm{~h}$

8 Severe electrolyte/acid-base abnormality (any of the following values):

(A) Sodium $<123$ or $>156 \mathrm{mmol} / \mathrm{l}$

(B) Potassium $<2.5$ or $>5.6 \mathrm{mmol} / \mathrm{l}$

(C) $\mathrm{HCO}_{3} \leq 14$ or $\geq 36 \mathrm{mmol} / \mathrm{l}$ (unless chronically abnormal)

(D) Arterial $\mathrm{pH}<7.30$ or $>7.45$

9 Sudden onset of unconsciousness or disorientation

10 Acute loss of sight or hearing within $48 \mathrm{~h}$

11 Acute loss of ability to move body parts within $48 \mathrm{~h}$

12 Persistent fever greater than $37.8^{\circ} \mathrm{C}$ orally or $38.3^{\circ} \mathrm{C}$ rectally for more than $48 \mathrm{~h}$ and where a diagnosis has not been established

13 Active bleeding

14 Wound dehiscence or evisceration

15 Pulse rate greater or less than the following ranges (optimally a sleeping pulse for $<12$ years old):

1 month to 6 months minus 1 day, $70-170 / \mathrm{min}$

6 months to 1 year minus 1 day, $80-160 / \mathrm{min}$

$2-6$ years of age, $70-160 / \mathrm{min}$

$7-11$ years of age, $60-160 / \mathrm{min}$

$>12$ years of age, $50-140 / \mathrm{min}$

16 Blood pressure values falling outside the following ranges:

6 weeks to 6 months minus 1 day, $70-110 \mathrm{~mm} \mathrm{Hg}$ (systolic)

6 months to 2 years minus 1 day, $70-100 / 40-85 \mathrm{~mm} \mathrm{Hg}$

2-6 years of age, $75-125 / 40-90 \mathrm{~mm} \mathrm{Hg}$

7-11 years of age, $80-130 / 45-90 \mathrm{~mm} \mathrm{Hg}$

$>12$ years of age, $90-150 / 60-120 \mathrm{~mm} \mathrm{Hg}$

17 Packed cell volume $<30 \%$

18 Need for lumbar puncture, where this procedure is not done routinely on an outpatient basis

19 Condition not responding to outpatient management (specify):

(A) Seizures

(B) Cardiac arrhythmia

(C) Bronchial asthma or croup

(D) Dehydration

(E) Persistent vomiting or diarrhea which needs inpatient assessment

(F) Abdominal pain which needs inpatient assessment

20 Special pediatric problems:

(A) Child abuse

(B) Noncompliance with the necessary therapeutic regimen

(C) Need for special observation or close monitoring of behavior including calorie intake in cases of failure to thrive 


\section{References}

$>1$ Liberati A, Apolone G, Lang T, Lorenzo S: A European project assessing the appropriateness of hospital utilization: background, objectives and preliminary results. Int J Qual Health Care 1995;7:187-199.

$>2$ Kreger BE, Restuccia JD: Assessing the need to hospitalize children: Pediatric Appropriateness Evaluation Protocol. Pediatrics 1989; 84:242-247.

3 Gertman PM, Restuccia JD: The appropriateness evaluation protocol: a technique for assessing unnecessary days of hospital care. Med Care 1981;19:855-871.

$\checkmark 4$ Kemper KJ: Medically inappropriate hospital use in a pediatric population. $\mathrm{N}$ Engl J Med 1988;318:1033-1037.
Restuccia JD: The evolution of hospital utilization review methods in the United States Int J Qual Health Care 1995;7:253-260.

6 Gloor JE, Kissoon N, Joubert GI: Appropriateness of hospitalization in a Canadian pe diatric hospital. Pediatrics 1993;91:70-74.

7 Formby DJ, McMullin ND, Danagher K, Oldham DR: The appropriateness evaluation protocol: application in an Australian children's hospital. Aust Clin Rev 1991;11:123131.

8 Katz M, Warshawsky SS, Porat A, Press J: Appropriateness of pediatric admissions to a tertiary care facility in Israel. Isr Med Assoc J 2001;3:501-503.
9 Bianco A, Pileggi C, Trani F, Angelillo IF: Appropriateness of admissions and days of stay in pediatric wards of Italy. Pediatrics 2003;112:124-128.

10 Esmail A, Quayle JA, Roberts C: Assessing the appropriateness of paediatric hospital admissions in the United Kingdom. J Public Health Med 2000;22:231-238.

11 Flores G, Abreu M, Chaisson CE, Sun D: Keeping children out of hospitals: parents' and physicians' perspectives on how pediatric hospitalizations for ambulatory care-sensitive conditions can be avoided. Pediatrics 2003;112:1021-1030.

12 Dougherty G: When should a child be in the hospital? A. Frederick North, Jr, MD, revisited. Pediatrics 1998;101:19-24. 